\begin{tabular}{|c|c|}
\hline Title & $\begin{array}{l}\text { Functional transformation series and the evolutionary origin of novel forms: evidence from a remarkable termite } \\
\text { defensive organ }\end{array}$ \\
\hline Author(s) & Kaji, Tomonari; Keiler, Jonas; Bourguignon, Thomas; Miura, Toru \\
\hline Citation & $\begin{array}{l}\text { Evolution and development, } 18(2), 78-88 \\
\text { https://doi.org/10.1111/ede.12179 }\end{array}$ \\
\hline Issue Date & 2016 \\
\hline Doc URL & http:/hdl. handle.net/2115/65035 \\
\hline Rights & $\begin{array}{l}\text { This is the peer reviewed version of the following article: http://onlinelibrary.wiley.com/doi/10.1111//ede.12179full, } \\
\text { which has been published in final form at } 10.1111 / \text { ede.12179. This article may be used for non-commercial purposes in } \\
\text { accordance with Wiley Terms and Conditions for Self-A rchiving. }\end{array}$ \\
\hline Type & article (author version) \\
\hline Additional Information & There are other files related to this item in HUSCAP. Check the above URL. \\
\hline File Information & Kaji et al MS CLEAN.pdf (本文) \\
\hline
\end{tabular}

Instructions for use 
Title:

\section{Functional transformation series and the evolutionary origin of novel forms: Evidence from a remarkable termite defensive organ}

\section{Running head:}

\section{Nasute termite evolution}

\section{author names and addresses:}

Tomonari Kaji

Bamfield Marine Sciences Centre, 100 Pachena Rd, Bamfield, BC Canada, V0R 1B0

flickloop@gmail.com

\section{Jonas Keiler}

Allgemeine und Spezielle Zoologie, Institut für Biowissenschaften, Universität Rostock jonas.keiler@gmx.de

Thomas Bourguignon Department of Biological Sciences, National University of Singapore thomas.bourgui@gmail.com

\section{Toru Miura}

Graduate School of Environmental Science, Hokkaido University miu@ees.hokudai.ac.jp

word count:

6808 words 


\begin{abstract}
The origins of evolutionary novelties are often deeply puzzling. They are generally associated with new functions that were absent in ancestors. The new functional configuration should arise via intermediate stages without any loss of function or impediment to the whole organism during the transitions. Therefore, understanding of the functional configurations of transitional states can shed light on how novel forms arise. Here we infer the evolutionary origin of a highly specialized termite defensive organ "nasus" where different functions overlap in different structural configurations at intermediate evolutionary stages to ensure that each phase is functional. Soldiers of a nasutitermitine termite use reconfigured mandibular muscles to squirt a viscous secretion from a nozzle-like head projection (the nasus). This contrasts sharply with the primitive defensive strategy where mandibles are used to bite. MicroCT observations of soldiers of Nasutitermes takasagoensis and of species with the ancestral state (Hodotermopsis sjostedti, Embiratermes neotenicus) revealed three different yet fully functional configurations in the transition from ancestral to novel state: 1) elevated hydrostatic pressure induced by contraction of mandibular muscles when biting gently oozes secretion from a gland, 2) direct pressure on an enlarged gland arises from expansion of the mandibular muscles when biting, 3) squirting in a piston-like manner by an inflated gland enveloped by highly modified mandibular muscles. Even a structure as exotic as the nasus therefore appears to have evolved with no loss of function at any stage. Such a functional approach holds much promise for understanding the evolutionary origin of seemingly preposterous novel forms.
\end{abstract}




\section{INTRODUCTION}

Evolutionary processes often yield striking new forms and functions, sometimes referred to as evolutionary novelties (Brigandt and Love 2012, Moczek 2008, Müller 2010). How such major morphological transformations occur between ancestral and novel states is often difficult to explain via gradual evolution (Frazzetta 1975, Müller and Wagner 1991, Futuyma 1998). The main challenge arises from the functional consequences of correlations among morphological parts, as noted by Cuvier (1826): “... all the organs of an animal form a single system, the parts of which hang together, and act and react upon one another; and no modifications can appear in one part without bringing about corresponding modifications in all the rest" (cited from Russell 1982: p.35). Although formulated in the 19th century Cuvier's dictum remains relevant in modern evolutionary biology (Mayr 1960, Amundson 2005) because novel forms presumably arise as a gradual transformation from ancestral states via intermediate stages that must be functional (Mayr 1960, Riedl 1975, Wake et al. 1983, Roth and Wake 1989, Raff 1996, Müller and Wagner 2003), even though functionality can be disrupted by elimination or alteration of any one of the interacting parts (Futuyma 1998, Schwenk 2001). Indeed, unveiling these intermediate steps of the evolution of novel forms can help explain how such transforming organs remain functional throughout the transformation (Kaji et al. 2011; Müller and Wagner 2003; Osorio 1994). Such studies show how the evolutionary steps of these organs are rationally elaborated to maintain functionality throughout the process. But difficulties remain because wide gaps still exist between the functional configurations of these assumed intermediate evolutionary stages, due to entirely new morphological elements that have no known function in the intermediate stages (e.g., "disc rim" and "circular muscles" in the novel suction discs of branchiuran crustaceans: Kaji et al. 2011). These gaps continue to raise questions about how transitions among functional configurations are overcome without a loss of functionality.

The striking novel organ of nasutitermitine termites, the "nasus", offers an intriguing new example to study the evolution of a highly derived novel form. The nasus is a caste-specific defensive organ possessed by soldiers in this subfamily: it is a nozzle-shaped frontal projection of the head that squirts a large volume of viscous, gluey secretion from a frontal pore (Deligne et al. 1981, Noirot 1969, Prestwich 1979, 1984, Scholtz et al. 2008) (Fig. 1). The secretion is stored in a huge frontal gland: an epithelial invagination from the frontal pore that expands extensively into the posterolateral sides of the head capsule. The frontal gland is a unique organ shared by several termite linages as showing in Figure $2 \mathrm{~B}$. The frontal gland is most developed in soldiers, but also occurs in workers and alate imagos (Kutalová et al. 2013, Šobotník et al. 2010a, c). The composition of secretions differs between taxa (Deligne et al. 1981, Prestwich 1984, Šobotník et al. 2010b), and consists of a toxic, viscous and sticky liquid that is squirted on enemies (Eisner et al. 1976, Noirot 1969, Nutting et al. 1974, Prestwich 1979). Modified mandibular muscles associated with inflated motor neurons are thought to contract the frontal gland to initiate squirting (Deligne et al. 1981, Ishikawa et al. 2008, Noirot 1969). This extraordinarily well-developed nasus gland complex, combined with extremely reduced mandibles (Miura et al. 1998, Sands 1957, Toga et al. 2011), yields a wholly novel functional morphology as a glue-squirting specialist called a "nasute" soldier ( $\mathrm{Na}$ in Fig. 2B).

In the several well studied genera of Nasutitermitinae (e.g. Nasutitermes and Hospitalitermes), soldiers develop from a "minor worker" via a "presoldier" stage (Miura et al. 1998) (Fig. 2A). The worker caste morphology is similar to the workers of other termite 
species, with well-developed mandibles and with no nasus. The morphogenesis of the nasus and the frontal gland starts in minor workers, just before they molt into presoldiers (Miura and Matsumoto 2000). In the presoldier stage, the nasus projection is already developed and connected to the reservoir of the frontal gland (Miura and Matsumoto 2000, Toga et al. 2012). Here, we describe the development of the frontal gland and the associated mandibular muscles.

A nasus-like structure appears to have evolved more than once. It is not unique to Nasutitermitinae, but also occurs in the Syntermitinae (Termitidae), sometimes called "mandibulate nasutes (MaNa in Fig. 2B)" because their soldiers possess both a nasus and biting mandibles. Traditionally, mandibulate nasutes were considered more closely related to true nasutes (Emerson 1949, Ahmad 1950). However, molecular phylogenies have shown that the nasutes and the mandibulate nasutes have independent origins (Inward et al. 2007, Scholtz et al. 2008, Bourguignon et al. 2015). Nonetheless, the enlarged biting mandibles and a nasus-less frontal gland in soldiers, is the ancestral condition in Nasutitermitinae, since the nasute is a monophyletic clade somehow derived from a mandibulate outgroup (Koovor 1969, Miller 1986, Prestwich and Collins 1981, Sands 1965, Seevers 1957) (see Fig. 2B). Although no taxa are known bearing intermediate forms between mandibulate and nasute soldiers, the presumed direction of evolution from mandibulate to nasute, is very similar to the developmental sequence of the nasute form. The development of the nasute form may therefore yield insights into its evolutionary origin, given the tendency for ontogeny to recapitulate phylogeny.

Here we report the results of our detailed study of the internal morphology of the head of soldiers of Hodotermopsis sjostedti (mandibulate form; Termopsidae), Embiratermes neotenicus (mandibulate nasute form; Termitidae) and Nasutitermes takasagoensis (fully nasute form; Termitidae) using microCT scanning techniques. Interspecific comparisons revealed that they have fundamentally discrete functional configurations to squirt the defensive secretion. Furthermore, detailed morphology of the developmental sequence of $N$. takasagoensis revealed a partially recapitulated evolutionary sequence of nasute morphology, including crucial observations of the likely functional relations of intermediate stages. Our observations suggest that two thresholds of functional configuration had to be overcome evolutionarily, but these thresholds were not crossed simultaneously. More significantly, each threshold appears to have been overcome without any loss of functionality at any stage. Finally, we propose a hypothesis that the evolutionary transition toward the fully-developed nasute state is a quasi-inevitable sequence resulting from the particular arrangement of muscles and tendons found in mandibulate ancestors.

\section{MATERIALS AND METHODS}

We studied three species: Embiratermes neotenicus (Termitidae) collected from Petit Saut, French Guiana; Hodotermopsis sjostedti (Termopsidae) from Yakushima Island, Kagoshima Prefecture, Japan; Nasutitermes takasagoensis (Termitidae) from Yaeyama Islands, Okinawa Prefecture, Japan.

In preparation for observations by microCT (Phoenix Nanotom-180; PhoenixjX-ray, GE Sensing \& Inspection Technologies, Wunstorf, Germany), specimens were fixed in either 
Bouins's solution or 70\% ethanol. Specimens were dehydrated in an ethanol series, freezedried with ES-2030 freeze dryer (Hitachi, Tokyo). Three-dimensional reconstructions were performed using Imaris 6.4.0 (Bitplane AG, Switzerland), and WinSURF (SURFdriver Software, Kailua, HI). Reconstructed images for figures were arranged with Adobe Photoshop CS5.

For transmission electron microscopy (TEM; Hitachi H-7500, Tokyo, Japan), living specimens were initially fixed in 2\% glutaraldehyde and 2\% paraformaldehyde in $0.1 \mathrm{M}$ cacodylate sodium buffer ( $\mathrm{pH} 7.4$ ) for $5 \mathrm{~h}$ at $4^{\circ} \mathrm{C}$; postfixed in $1 \%$ osmium tetroxide in the same buffer with $5 \%$ sucrose for $2 \mathrm{~h}$ at $4^{\circ} \mathrm{C}$; dehydrated through a graded acetone series; and then embedded in Spurr's resin and polymerized. Sections were obtained using an ultramicrotome and stained with a $1 \%$ potassium permanganate solution in distilled water for 2 min followed by $2 \%$ lead citrate for $3 \mathrm{~min}$.

\section{RESULTS}

Musculoskeletal system in the head of "mandibulate" soldiers of Hodotermopsis sjostedti

The mandible closer-muscles originate from ventral, posterior, lateral and dorsal parts of the head capsule (Fig. 3A, B) and insert in the median edge of the mandibles via a tendon (Fig. 3C). Each muscle has two lobes on the tendon (inner lobe and outer lobe: Fig. 3D). The muscles can be divided into several "bundles" which are composed of units of muscle fibers. Here, we establish an operational segmentation of bundles defined by their attached face to the lobes on the tendon (af1-5 in Fig. 3D). According to our operational definition, $H$. sjostedti possess five bundles. Bundle 1 (bn1) originates on the dorsal area of the head capsule and inserts on attachment face 1 of the tendonous lobe (af1). Bundle 2 (bn2) originates on the dorsal area of the head capsule and inserts on attachment face 2 of the tendonous lobe (af2). Bundle 3 (bn3) originates on the dorsolateral area of the head capsule and inserts on attachment face 3 of the tendonous lobe (af3). Bundle 4 (bn4) originates on the ventral area of the head capsule and inserts on attachment face 4 of the tendonous lobe (af4). Bundle 5 (bn5) originates on the posterior end of the head capsule and inserts on attachment face 5 of the tendonous lobe (af5). A frontal gland is absent in this species.

Musculoskeletal system and frontal gland in the head of "mandibulate nasute" soldiers of Embiratermes neotenicus

The muscular configuration in this species is quite similar to $H$. sjostedti: five bundles form the mandible closer muscle; they originate from the ventral, posterior, lateral and dorsal parts of the head capsule (Fig. 4A) and insert on the median edge of the mandibles via tendons (Fig. 4D). The nasus projects from the anterior of the head capsule (Fig. 4A, B). The frontal gland invaginates from the frontal opening of the nasus and extends to the middle portion of the head capsule. The posterior part of the frontal gland gets pinched between the left and right bundle 1(Fig. 4A-C).

Developmental transformation from mandibulate worker to nasute soldier in Nasutitermes takasagoensis

Minor worker (mandibulate) (Fig. 5A, B): The mandible closer muscles originate from ventral, posterior, lateral and dorsoposterior parts of the head capsule and insert in the median edge of the mandibles via tendons. As in $H$. sjostedti and E. neotenicus, muscle bundles 1-5 
are recognized in this species as well. They have a similar configuration to $H$. sjostedti and $E$. neotenicus, but bundle 2 appears to be divided into several/more units. Although the soldier's head capsule is almost fully occupied by the mandible closer muscles, in the minor worker of $N$. takasagoensis the closer muscles only occupy half of the space and thereby leave open spaces (hemocoel) around the brain. The tentorial-fontanellar muscles originate from the epithelial tissue just behind the brain and insert in the tentorium (Fig. 5A, 6A). A frontal gland was not clearly visible in minor workers because of the resolution of microCT, but as described by Šobotník et al. (2010c), it occurs at the dorsal base of the tentorial-fontanellar muscles.

Early presoldiers (Fig. 5C, D): The configurations of the mandible closer muscles are conserved as in minor workers, but the gap between the left and right mandible closermuscles is widely opened, and these bundles are slightly flattened along the anteroposterior axis. The nasus is already fully developed and the frontal gland is invaginated from the tip of the nasus and elongates into the gap between left and right mandible closer muscles. The inner lobes of the tendons are slightly flattened and enlarged along the sagittal axis. Tentorialfontanellar muscles are thicker than in the minor workers. The frontal gland is dorsoventrally stretched (Fig. 6B). The dorsal side of the frontal gland is connected to the exoskeleton of the head capsule by two stripes of muscle-like tissue (arrow heads in Fig. 6B, C).

Late presoldiers (Fig. 5E, F): The posterior end of the frontal gland expands into the gap between bundles 1 and 5 . These bundles are drastically modified to allow the expansion of the gland: bundles 1 and 5 are flattened laterally to put the frontal gland between them, and bundle 2 gets flatter to wrap the expansion of the gland from both lateral sides. The inner lobes of the tendons are also modified to wrap the expanded gland from ventral side. Bundles 3 and 4 have also become flattened along with bundle 2 .

Nasute soldiers (Fig. 7): The nasus has become more pointed/cuspidate and the head capsule has distinctly expanded and possesses a rotund shape. The frontal gland is greatly expanded. Bundles 1 and 5 envelop the frontal gland and pinch the gland mostly along the anteroposterior axis (Fig. 7A,B: note that the gland is artificially shrunken due to the freeze drying process). Bundle 2 connects the transformed tendon (tendonous diaphragm, td) to the lateral circumference of the head capsule (Fig. 7C-E). The tendonous diaphragms show a sheet-like cuticular layer covering the frontal gland and bundles 1 and 5 (Fig. 7C-E). The bundles 1 and 5 attach to the diaphragms at an angle of approximately 70 degrees, giving a piston-like shape (Fig. 7E, 8A). The tendonous diaphragm is formed by two sub-layers of the cuticular sheet (Figs. 8A-C). The two-layered structure is observed at the inner lobe of the mandible closing tendon in minor workers (Figs. 8D). Each single bundle may correspond to a sublayer of the tendons, i.e., bundles 2 and 5 correspond to sublayers 2 and 5 of the tendonous diaphragm in the soldiers (Fig. 8C), bundles 1 and 2 correspond to sublayers 1 and 2 of the tendon in minor workers (Fig. 8D). Vestigial tendons are derived from the middle portion of the tendonous diaphragms and project toward the median edge of the mandibles (Fig. 7E), but the tendons are likely disconnected from the mandibles. Muscle bundles 3 and 4 are not observed in this stage. Tentorial-fontanellar muscles are still visible, remaining in the same place as in presoldiers (Fig. 6D).

\section{DISCUSSION}

Homology of muscles: The extremely modified 0md1 muscle operates squirting in nasute 
$\underline{\text { soldiers }}$

Although the results section referred to morphological characters with a homology-free terminology (morphemes sensu Richter and Wirkner 2014), we can infer homology throughout the examined taxa based on the connectivity (special arrangement) between morphological elements.

Wipfler et al. (2011) termed the mandible closer muscle present in the dicondylian arthropods (Zygentoma + Pterygota) as "Musculus craniomandibularis internus (0md1)", which is applicable for the studied termites. Herein described bundles 1-5 can be interpreted as homologous units among these species due to their similar distinct arrangement and correspondence of their attachment to the tendonous lobe. This subdivided nature of 0md1 and tendonous lobes occurs commonly in termites, and moreover, in Dicondylia (Blanke et al. 2012, Matsuda 1965, Vishnoi 1956, 1962, Wipfler et al. 2011).

The interpretation of homology can be extended to nasute soldiers using ontogenetic data of Nasutitermes takasagoensis. The homologies of mandible closing muscles ( $=0 \mathrm{md} 1)$ and each of the described bundles bn1-5 are traceable from minor worker to presoldier, since their arrangement transforms gradually with no apparent alteration or deletion of muscular substructures (Fig. 5). Despite their drastic transformation (Fig. 7), 0md1 muscles can also be identified in the nasute soldier, based on their origins (posterior, lateral and dorsal parts of the head capsule) and insertion points (median edge of the mandible). Bundles 1 and 5 are identical as the homologues to the same numbered bundles in earlier developmental stages, because of the connectivity between these muscles and the head capsule. We interpret the highly specialized tendon called the "tendonous diaphragm" as the homologue of the inner lobe in a presoldier, according to their connectivity with the muscle bundles 1 and 5 . Therefore bundle 2 in a nasute soldier could be homologous to the same numbered bundle in a presoldier, because the inner lobe should be sandwiched between bundles 1, 5 and 2 (see Figs. 3D and 7E). The outer lobes and their related muscles (bn3-4) presumably degenerate after the "late presoldier" stage, because we did not observe any evidence of fusion between the inner and outer lobes during their development.

\section{Distinct functional configurations and their evolutionary sequence}

Given the above differences in the squirting process, we identify here three distinctive categories of functional configurations (Fig. 9). Functional configuration (1) is a hydrostaticpressure type, where a small sac of the frontal gland is emptied by increased body pressure induced incidentally by the contraction of intersegmental muscles ("mandibulate phase", Fig. 9; the mechanism suggested by Šobotník et al. 2010a). Although some of the basal mandibulate species do not have a frontal gland, other mandibulate species do (Fig. 2B). Such a mandibulate phenotype with a small (but functional) frontal gland should represent this category of functional configuration. Since the mandibulate phenotypes we examined (soldier of Hodotermopsis sjostedti and minor worker of Nasutitermes takasagoensis) should have a vestigial frontal gland under the exoskeleton, they also could be considered to have a hydrostatic-pressure type functional configuration.

Functional configuration (2) is a muscular-pinching type, where a sac of the frontal gland extends to a point almost halfway between the pair of mandible closer-muscles (bn1, blue) and is directly in contact with and pinched between them (orange arrows, Fig. 9). The contraction of the bn 1 to close the mandibles increases the diameters of bn 1 which then pinch 
the sac of the frontal gland and may result in squirting (see Fig. 4B, C and "intermediate phase" in Fig. 9). Mandibulate nasutes and also mandibulate phenotypes that have an elongated frontal gland fall into this category. The flattened and enlarged shape of bn1 in Embiratermes neotenicus (Fig. 4A) indicates that the bundle controls the pinching function. The early presoldier of Nasutitermes takasagoensis (Fig. 5C, D) likely represents a recapitulated phase of the muscular-pinching type, even though the frontal gland is not yet functional. The species that exhibit the muscular-pinching type configuration likely retain some hydrostatic pressure effects because body pressure may affect the frontal gland anyway.

Functional configuration (3) is a piston type, where the frontal gland is expanded and squeezed between muscle bundles bn 1 and bn5, where the tendonous diaphragm covers the ventral side and muscle bundle bn2 connects the diaphragm to the lateral wall of the head capsule. This configuration - high attachment angle of the muscle bundles bn1 and bn5 to the tendonous diaphragm without any compensating bundles on the ventral side (see Fig. 7E and "nasute phase" in Fig. 9) - strongly suggests that the tendonous diaphragm works like a piston to generate the squirt. The curvatures of the muscle fibers of bundles 1 and 5 along with the frontal gland further suggest that these bundles also partially pinch the frontal gland as in the muscular-pinching type configuration.

Each of these three functional configurations squirts the secretion by a totally different mechanism. Large gaps between functional configurations raise challenging questions about how such gaps are overcome without any loss of correlation among parts during evolutionary transitions. Phylogenetic relations among the various anatomical phases are essential to understanding the evolutionary history of functional changes. The mandibulate-soldier form, which basically has a hydrostatic-pressure type configuration (Mandibulate phase, Fig. 9), is definitely the ancestral phase (Fig. 2B). Somewhat surprisingly, the extant mandibulatenasute soldier form, which represents a muscular-pinching type configuration (Intermediate phase, Fig.9), appears to have evolved independently from this ancestral state and is not likely the sister group to the fully-developed nasute-soldier form (Fig. 2B; Ohkuma et al. 2004, Scholtz et al. 2008). However, a fully developed nasus is not a functional requirement for the muscular-pinching type configuration, since that configuration is defined by connections between the gland and the mandibular muscles, not by an enlarged nasus. Furthermore, such a pinching configuration occurs in several mandibulate-soldier lineages that have an enlarged sac-like frontal gland but lack a nasus. For example, the frontal gland sometimes expands towards the posterior side of the head, and sometimes reaches the abdomen in the mandibulate-soldier caste of Rhinotermitidae and Serritermitidae (Šobotník et al. 2010a). In these cases the frontal gland attaches to or lies between the left and right mandible closer-muscles. These mandibulate-soldier castes with enlarged frontal glands could definitely be categorized as lineages that have the muscular pinching type configuration. On the other hand, a little nasus-like protuberance around a fontanelle (opening of the frontal gland) does occur in some linages of mandibulate soldiers (Mafp in Fig. 2B). This variability of frontal projections suggests that multiple mandibulate lineages have the potential to produce some sort of "intermediate" morphology towards the mandibulate-nasute soldier descendant. The phylogenetic relationship between such "muscular pinching" mandibulatesoldier lineages (that have an enlarged gland) and the nasute-soldier linage was not reported, but we suggest that the direct ancestor of the fully nasute form had a developed frontal gland that was pinched by muscles, because as we have shown at the developmental intermediate of nasute soldier (early presoldier, Fig. 5) the gland cannot achieve the "nasute" position without first achieving the pinching position, otherwise evolution must have occurred by saltation. 
Gradual evolution yields saltational acquisition of new functional configurations

We propose a hypothetical evolutionary sequence for the remarkable transition from the ancestral mandibulate form to the highly derived, fully nasute form in light of the above functional configurations. From the mandibulate phase to the hypothetical intermediate phase, the frontal gland simply elongated posteriorly until it extended into the cleavage between muscles (see Fig. 9; see also Fig. 4A, B). During gland elongation, elevated hydrostatic pressure (black arrows, Fig. 9) was the primary cause of squirting, but the pinching configuration (orange arrows, Fig. 9) was achieved immediately after the gland reached and extended into the cleavage between muscles. In this phase, the transition between functional configurations would have been complete once the physical threshold between the gland's muscle-free position ("mandibulate phase", Fig. 9) and the gland's musclular-pinching position ("intermediate phase", Fig. 9) was overcome. Despite the punctuated nature of the functional configuration, the morphological transformation itself (elongation of the frontal gland) was quite gradual and simple.

The transition from the intermediate phase to the nasute phase was more complex (Fig. 9). In this transition, the frontal gland expanded between muscle bundles 1 and 5 (red and blue, "nasute phase", Fig. 9) and pinching was greatly enhanced through additional pinching contributed by muscle bundle 5 (red). Finally, as the gap between bundles got wider, the simple tendons transformed into a tendonous diaphragm (yellow) to make the overall structure resemble a piston. Such a transformation of the functional configuration would have been achieved once the tendon became thin enough to behave as a diaphragm, and the separation between bundles was wide enough to allow the tendonous diaphragm to buckle. So the transition to the piston-type configuration relied on the degree of thickness of the tendon and the separation between muscle bundles. In this transformational process also, the morphological transformation itself was gradual but the transformation between functional configurations was punctuated. Significantly, these functional thresholds between mandibulate and nasute phases were overcome without any loss of overall functionality of the head, because primitive functions overlapped more derived functions in each phase. Namely, the primary hydrostatic pressure effects in the mandibulate phase (black arrows) carried over to the intermediate phase, and the lateral pinching effect in the intermediate phase (orange arrows) also carried over to the nasute phase (see Fig. 9). Therefore, the evolution of the dramatic nasute soldier phase could occur without any loss of overall functionality at any stage during transitions.

Notably, these elaborate evolutionary changes in functional configurations were achieved by limited morphological elements - bundles and tendons of mandible closer-muscles, frontal gland and fontanelle — that already existed in the mandibulate ancestors. Therefore, the morphological arrangement in the ancestor could be interpreted as a "precondition" for the evolution of the fully nasute state via fully functional intermediates. Namely, the transition from the hydrostatic configuration to the pinching configuration (Fig. 9) could occur only if the ancestor had a pair of mandibular closer-muscles, and the transition from the pinching configuration to the piston configuration could occur only if the ancestor had a sheet of tendon underneath properly subdivided bundles to enclose the expanding gland. Such preconditions, especially subdivided tendonous lobes and bundles, are shared by every hexapod with biting mandibles (Blanke et al. 2012, Matsuda 1965, Wipfler et al. 2011). Muscles that have such subdivided bundles and tendons are called "multipennate muscles", and are commonly found in animals (Dorit et al. 1991). Multipennate structure makes it possible to contract the application point in various ways to produce more flexible motion 
(Larson and Stern 2007). The multipennate muscle-type may have existed in the stem lineage of hexapod to permit flexible operation of the mandibles, and may have later been diverted as an exaptation (sensu Gould and Vrba 1982) to achieve the novel form of the nasute termite as a quasi-inevitable result. In other words, the preconditions for the dramatic evolutionary transformations to the nasus form were all already present in the ancestor. But these deterministic steps were only achieved via the appropriate elongation and expansion of the frontal gland, which were driven by a completely unknown mechanism.

The evolution of the termite nasus form described here adds to the small but fascinating collection of well-documented examples where wholly novel forms evolved via rearrangement of preexisting morphological elements, including the venom delivery system in centipedes (Dugon and Arthur, 2012, Dugon et al. 2012) and copepods (Ohtsuka et al. 1997, Nishida and Ohtsuka 1996), and the hindlimbs of birds (Müller and Streicher 1989). It also affirms the value of a functional approach to a better understanding of the origin of evolutionary novelties. It can demystify transitions between seemingly incommensurable states by revealing functional intermediates, the lack of which has led some to suggest that the evolutionary origin of novelty is qualitatively different from conventional evolution.

\section{Acknowledgments}

The authors express deep gratitude to Dr. Kiyoto Maekawa and Mr. Shutaro Hanmoto (University of Toyama) for providing the specimens of $N$. takasagoensis. Deep thanks are given also to Dr. Stefan Richter and Dr. Christian Wirkner (Universität Rostock) for their support and usage of their microCT. T.K. is very appreciative of Dr. Richard Palmer (University of Alberta) for continuous encouragement and substantial English editing on the paper. We are deeply grateful to Dr. Benjamin Wipfler and an anonymous referee for critical reading and constructive suggestions. Deep thanks are also extended for valuable suggestions from Dr. Rolf Beutel (Friedrich-Schiller-Universität Jena). T.K. also acknowledges Dr. Yuta Shiino (Niigata University) and Dr. Yutaro Suzuki (Shizuoka University) for valuable discussion on the concept of functional morphology. T.K. is grateful to B.Sc. Neil Waters for improving the English of this paper. 


\section{REFERENCES}

Amundson, R. 2005. The Changing Role of the Embryo in Evolutionary Thought: Roots of Evo-Devo. Cambridge University Press, New York.

Beutel, R. G., Zimmermann, D., Krauß, M., Randolf, S., and Wipfler, B. 2010. Head morphology of Osmylus fulvicephalus (Osmylidae, Neuroptera) and its phylogenetic implications. Org. Divers. Evol. 10: 311-329.

Blanke, A., Wipfler, B., Letsch, H., Beutel, R.G., and Misof, B. 2012. Revival of Palaeoptera - head characters support a monophyletic origin of Odonata and Ephemeroptera (Insecta). Cladistics 28: 560-581.

Bourguignon, T., and Roisin, Y., 2011. Revision of the termite family Rhinotermitidae (Isoptera) in New Guinea. ZooKeys 148: 55-103.

Bourguignon, T., Lo, N., Cameron, S. L., Šobotník, J., Hayashi, Y., Shigenobu, S., \& Evans, T. A. 2015. The evolutionary history of termites as inferred from 66 mitochondrial genomes. Mol. Biol. Evol. 32: 406-421.

Brigandt, I., and Love, A. 2012. Conceptualizing Evolutionary Novelty: Moving Beyond Definitional Debates. J. Exp. Zool. (Mol. Dev. Evol.) 318B: 417-427.

Deans, A. R., Mikó, I., Wipfler, B., and Friedrich, F., 2012. Evolutionary phenomics and the emerging enlightenment of arthropod systematics. Invertebrate systematics 26: 323-330.

Deligne, J., Quennedey, A., and Blum, M. S., 1981, The enemies and defense mechanisms of termites. In H. R. Hermann (ed.). Social insects Volume 2, Academic Press, New York, pp. 176.

Dorit, R. L., Walker, W. F. Jr., and Barnes, R. D. 1991. Zoology. Saunders College Publishing, Philadelphia.

Dugon, M. M., and Arthur, W. 2012. Comparative studies on the structure and development of the venom-delivery system of centipedes, and a hypothesis on the origin of this evolutionary novelty. Evol Dev 14: 128-137.

Dugon, M. M., Hayden, L., Black, A., and Arthur, W. 2012. Development of the venom ducts in the centipede Scolopendra: an example of recapitulation. Evol Dev 14: 515-521.

Eisner, T., Kriston, I., and Aneshansley, D. J. 1976. Defensive behavior of a termite (Nasutitermes exitiosus) (Isopt. Termitidar). Behav. Ecol. Sociobiol 1: 83-125.

Emerson, A. E. 1971. Tertiary fossil species of the Rhinotermitidae (Isoptera), phylogeny of genera, and reciprocal phylogeny of associated Flagellata (Protozoa) and Staphylinidae (Coleoptera). B. Am. Mus. Nat. Hist. 146: 143-303.

Futuyma, D. J. 1998. Evolutionary Biology, 3rd edition. Sinauer, Massachusetts. p. 763. 
Frazzetta, T. H. 1975. Complex adaptations in evolving populations, Snauer, Massachusetts. p. 267.

Cuvier G. 1826. Histoire des progrès des sciences naturelles : depuis 1789 jusqu'à ce jour. Baudouin Freres, Paris.

Gould, S. J., and Vrba, E. S. 1982. Exaptation: A missing term in the science of form. Palaeobiology 8: 4-15.

Inward, D. J. G., Vogler, A. P., and Eggleton, P. 2007. A comprehensive phylogenetic analysis of termites (Isoptera) illuminates key aspects of their evolutionary biology. Mol. Phylogenet. Evol. 44: 953-967.

Ishikawa, Y., Aonuma, H., and Miura, T. 2008. Soldier-specific modification of the mandibular motor neurons in termites. PLoS ONE 3: e2617.

Kaji, T., Møller, O. S., and Tsukagoshi, A. 2011. A bridge between original and novel states: ontogeny and function of "suction discs" in the Branchiura (Crustacea). Evol. Dev. 13: 119126.

Koovor, J. 1969. Anatomie comparée du tube digestif des termites II. Sous-famille des nasutitermitinae. Insectes Soc. 16: 195-234.

Kutalová, K., Bourguignon, T., Dussès, D. S., Hanus, R., Roisin, Y., and Šobotník, J. 2013. Armed reproductives: Evolution of the frontal gland in imagoes of Termitidae. Arthropod Struct. Dev. 42: 339-348.

Larson, S. G, and Stern, J. T. Jr. 2007. Humeral retractor EMG during quadrupedal walking in primates. J. Exp. Biol. 210: 1204-1215.

Matsuda, R. 1965. Morphology and evolution of the insect head. Memoirs of the American Entomological Institute 4: 1-334.

Mayr, E. 1960 In Evolution after Darwin, ed Tax S. University of Chicago Press, Chicago. pp 349-380.

Moczek, A. P. 2008. On the origins of novelty in development and evolution. Bioessays 30: 432-447.

Miller, L. R. 1986. The phylogeny of the Nasutitermitinae (Isoptera: Termitidae). Sociobiology 11: 203-214.

Miura, T., and Matsumoto, T. 2000. Soldier morphogenesis in a nasute termite: discovery of a disc-like structure forming a soldier nasus. Proc. R. Soc. Lond. B Biol. Sci. 267: 1185-1189.

Miura T, Roisin Y, Matsumoto T. 1998. Developmental Pathways and Polyethism of Neuter Castes in the Processional Nasute Termite Hospitalitermes medioflavus (Isoptera: Termitidae). Zoological Science 15: 843-848. 
Müller, G. B., and Streicher, J. 1989. Ontogeny of the syndesmosis tibiofibularis and the evolution of the bird hindlimb: A caenogenetic feature triggers phenotypic novelty. Anatomy and Embryology 179: 327-339.

Müller, G. B., and Wagner, G. P. 2003. Innovation. In B. K. Hall, M. N. Olson (eds.) Keywords and concepts in evolutionary developmental biology. Harvard University Press, Cambridge MA. pp. 218-227.

Müller, G. B. 2010. Epigenetic Innovation. In M. Pigliucci and G. B. Müller (eds.). Evolution - the Extended Synthesis. MIT press, Cambridge. pp. 307-333.

Nishida, S., Ohtsuka, S. 1996. Specialized feeding mechanism in the pelagic copepod genus Heterorhabdus (Calanoida: Heterorhabdidae), with special reference tothe mandibular tooth and labral glands. Mar. Biol. 126: 619-632.

Noirot, C. H. 1969. Glands and secretions. In K. Krishna and F. M. Weesner (eds.). Biology of Termites. Academic Press, New York. pp. 89-123.

Nutting, W. L., Blum, M. S., and Fales, H. M. 1974. Behaviour of the North American termite Tenuirostritermes tenuirostris, with special reference to the soldier frontal gland secretion, its chemical composition and its use in defense. Psyche 81: 167-177.

Ohkuma, M., Yuzawa, H., Amornsak, W., Sornnuwat, Y., Takematsu, Y., Yamada, A., Vongkaluang, C., Sarnthoy, O., Kirtibutr, N., Noparatnaraporn, N., Kudo, T., and Inoue, T. 2004. Molecular phylogeny of Asian termites (Isoptera) of the families Termitidae and Rhinotermitidae based on mitochondrial COII sequences. Mol. phylogenet. Evol. 31: 701710.

Osorio, D. 1994. Eye evolution: Darwin's shudder stilled. Trends in Ecology and Evolution 9: 241-242.

Ohtsuka, S., Soh, H. Y., and Nishida, S. 1997. Evolutionary switching from suspensionfeeding to carnivory in the calanoid family Heterorhabdidae (Copepoda). J. Crust. Biol. 17: 577-595

Prestwich, G. 1979. Chemical defense by termite soldiers. Journal of Chemical Ecology 5: 459-480.

Prestwich, G. D. 1984. Defense mechanisms of termites. Annu Rev Entomol 29: 201-232.

Prestwich, G. D., Collins, M. S. 1981. Chemotaxonomy of Sublitermes and Nasutitermes termite soldier defense secretions. Evidence against the hypothesis of diphyletic evolution of the Nastutitermitinae. Biochem. System. Ecol. 9: 83-88.

Russell, E. S. 1982. Form and Function: A Contribution to the History of Animal Morphology. University of Chicago Press, Chicago. p. 383.

Raff, R. A. 1996. The shape of life. Gene, development, and the evolution of animal form. University of Chicago Press, Chicago. p. 544. 
Richter, S., and Wirkner, C. S. 2014. A research program for Evolutionary Morphology. J Zoolog Syst Evol Res. 52: 338-350.

Riedl, R. 1975. Die Ordnung des Lebendigen. Systembedingungen der Evolution. Paul Parey Verlag, Hamburg. p. 372.

Roth, G., and Wake, D. B. 1989. Conservatism and Innovation in the Evo- lution of Feeding in Vertebrates. In D. B. Wake and G. Roth (eds.). Complex Organismal Functions: Integration and Evolution in Vertebrates. Jofn Wiley and Sons, Chichester, pp. 7-21.

Sands, W. A. 1957. The soldier mandibles of the Nasutitermitinae (Isoptera, Termitidae). Insectes Soc. 4: 13-24.

Sands, W. A. 1965. A revision of the terrmite subfamily Nasutiter- mitinae (Isoptera, Termitidae) from the Ethiopian region. Bull. Br. Mus. Nat. Hist. Suppl. 4: 1-172.

Seevers, C. H. 1957. A monograph on the termitophilous Staphylin- idae (Coleoptera). Fieldiana 40: 1-334.

Scholtz, O. I., MacLeod, N., and Eggleton, P. 2008. Termite soldier defence strategies: A reassessment of Prestwich's classification and an examination of the evolution of defence morphology using extended eigenshape analyses of head morphology. Zool. J. Linn. SocLond. 153: 631-650.

Schwenk, K. 2001. Functional units and their evolution. In: G. P. Wagner (ed.). The Character Concept in Evolutionary Biology. Academic Press, San Diego. pp. 165-198.

Šobotník, J., Bourguignon, T., Hanus, R., Sillam-Dussès, D., Pflegerová, J., Weyda, F., Kutalová, K., Vytisková, B., Roisin, Y. 2010. Not Only Soldiers Have Weapons: Evolution of the Frontal Gland in Imagoes of the Termite Families Rhinotermitidae and Serritermitidae. PLoS ONE 5: e15761.

Šobotník, J., Jirošová, A., Hanus, R. 2010. Chemical warfare in termites. J. Insect Physiol. 56: 1012-1021.

Sobotník, J., Sillam-Dussès, D., Weyda, F., Dejean, A., Roisin, Y., Hanus, R., and Bourguignon, T. 2010. The frontal gland in workers of neotropical soldierless termites. Naturwissenschaften 97: 495-503.

Toga, K., Hojo, M., Miura, T., and Maekawaa, K. 2012. Expression and function of a limbpatterning gene Distal-less in the soldier-specific morphogenesis in the nasute termite Nasutitermes takasagoensis. Evol. Dev. 14:3 286-295.

Toga, K., Yoda, S., and Maekawa, K. 2011. The TUNEL assay suggests mandibular regression by programmed cell death during presoldier differentiation in the nasute termite Nasutitermes takasagoensis. Naturwissenschaften 98: 801-806.

Vishnoi, H. S. 1956. The Structure, Musculature and Mechanism of the Feeding Apparatus of the various castes of the termite Odontotermes obesus (Rambur). Part I. Clypeo-labrum. 
Journal of the Zoological Society of India, 8: 1-18.

Vishnoi, H. S. 1962. Musculature of a common Indian termite Odontotermes obesus (Rambur)(Isoptera, Termitidae). Bull Entomol (Loyola College, Madras) 3: 13-20.

Wake, D. B., Roth, G., Wake, M. H. 1983. On the problem of stasis in organismal evolution. J. Theor. Bio. 101: 211-224.

Weihmann, T., Gorb, B., Kleinteich, T., and Wipfler, B. (in press) Functional morphology of the chewing apparatus in the cockroach Periplaneta americana (Blattodea, Blattidae) - A role model for generalist omnivore insects. Arthropod Systematics and Phylogeny, in press.

Wipfler, B., Machida, R., Müller, B., and Beutel, R. G. 2011. On the head morphology of Grylloblattodea (Insecta) and the systematic position of the order, with a new nomenclature for the head muscles of Dicondylia. Systematic Entomology 36: 241-266. 


\section{FIGURE LEGENDS}

Fig. 1. Squirting by a nasute soldier of Nasutitermes takasagoensis. (A) Squirting started immediately after provocation by an ant's leg. (B) 0.038 second after (A). (C) 0.074 second after (A), just after squirting was finished. After ejection, the volume of secreted material shrank and thickened into viscous glue. Simultaneously the $N$. takasagoensis step backward to escape from the enemies until the secretion is replenished.

Fig. 2. Ontogeny and phylogeny of morphological states of the mandibles, frontal glands and nasus. (A) Ontogeny of nasute soldier, adopted from Miura et al. (1998). (B) Simplified phylogeny of termites at family and subfamily levels, based on Bourguignon et al. (2015). Typical morphological states of solders are illustrated. Mandibulate types in this figure include several types of defense morphology as described by Prestwich (1984). Morphological states are adopted from Bourguignon and Roisin (2011), Prestwich (1984) and Scholtz et al. (2008). Ma, mandibulate type; Maf, mandibulate type with frontal gland; Mafp, mandibulate type with frontal gland and protuberant fontanelle; MaNa, mandibulate nasute; $\mathrm{Na}$, nasute.

Fig. 3. Three-dimensional microCT reconstruction of mandible closer muscles and exoskeleton in a "mandibulate" soldier of Hodotermopsis sjostedti. (A) Posterodorsal view of the right side of the head. (B) Anteroventral view of the head. (C) Posterodorsal view showing a coronal plane. (D) Posterolateral view (right side) of the tendons belonging to mandible closer muscles. af1-5, attachment face of muscle bundles 1-5 in the tendonous lobe; bn1-5, bundles 1-5 of mandible closer muscles. Scale bars, $1 \mathrm{~mm}$.

Fig. 4. Three-dimensional microCT reconstruction of mandible closer muscles, frontal gland and exoskeleton in a "mandibulate nasute" soldier of Embiratermes neotenicus. (A) Posterodorsal view of the right side of the head. (B) Posterodorsal view showing a coronal plane. (C) Posterolateral view (right side) showing a transverse plane. (D) Posterodorsal view showing a coronal plane. bn1-5, bundles 1-5 of mandible closer muscles. Scale bars, $500 \mu \mathrm{m}$.

Fig. 5. Three-dimensional microCT reconstruction of mandible closer muscles, frontal gland, tentorial-fontanellar muscle and exoskeleton in different developmental stages of Nasutitermes takasagoensis. (A) Posterodorsal view of the head of a minor worker. (B) Posterodorsal view of a minor worker showing both a coronal and transverse plane. (C) Posterodorsal view of an early presoldier. (D) Posterodorsal view of an early presoldier showing a coronal plane. (E) Posterodorsal view of a late presoldier. (F) Posterodorsal view of a late presoldier showing a coronal plane. b, brain; bn1-5, bundles 1-5 of mandible closer muscles; tfm, tentorial-fontanellar muscle. Scale bars, 100 $\mu \mathrm{m}$.

Fig. 6. Three-dimensional microCT reconstruction of the tentorial-fontanellar muscle in developing Nasutitermes takasagoensis. (A) Anterolateral view showing a transverse plane of the head in a minor worker. (B) Anterolateral view showing a sagittal plane in a presoldier. (C) Anterolateral view showing a transverse plane in a presoldier. (D) Anterolateral view showing a transverse plane in a soldier. Arrowhead, muscle-like tissue; b, brain; fg, frontal gland; p, pharynx; sg, suboesophageal ganglion; te, tentorium; tfm, tentorial-fontanellar muscle. Scale bars, $100 \mu \mathrm{m}$. 
Fig. 7. Three-dimensional microCT reconstruction of mandible closer muscles, frontal gland and exoskeleton in a "nasute" soldier of Nasutitermes takasagoensis. (A) Posterodorsal view of the head. (B) Anteroventral view, slightly from right side. (C) Posterodorsal view showing a coronal plane. (D) Anteroventral view showing a coronal plane. (E) Lateral view (right side) showing a sagittal plane. an, antenna; bn1-5, bundles 1-5 of mandible closer muscles; fg, frontal gland; lp, labial palp; mp, maxillary palp; td, tendonous diaphragm; vt, vestigial tendon. Scale bars, $100 \mu \mathrm{m}$.

Fig. 8. Transmission electron microscopy of mandible closer tendons in Nasutitermes takasagoensis. (A) Sagittal section of the tendonous diaphragm and muscles in a nasute soldier. The area corresponds to the indicated circle in fig. 7E. (B) sagittal section of the tendonous diaphragm and frontal gland in a nasute soldier. The area corresponds to the indicated circle in fig. 7E. (C) Magnified tendonous diaphragm. (D) Tendons and muscles of a minor worker. bn1-5, bundles 1-5 of mandible closer muscles; td, tendonous diaphragm; sec, secretory cells of frontal gland; sl-ten1-2, sub-layers 1-2 of the tendon; sl-td2-5, sub-layers 25 of tendonous diaphragm. Scale bars, $500 \mathrm{~nm}$ for A, 200nm for B-D.

Fig. 9. Transformation of functional configurations during nasute evolution. The illustration only shows the connectivity between the inner lobes and related muscle bundles and frontal gland. Muscle colors indicate specific muscle bundles: blue- bn1, green- bn2, red- bn5, yellow- tendon. Arrows indicate squirting forces directed towards the frontal gland: blackincidental hydrostatic forces; orange- direct muscle action on the frontal gland; purple- direct pumping action on the frontal gland from tendonous diaphragm; arrow size indicates relative size of forces that contribute to squirting. Large arrow colors and backgrounds show what functions are present at various intermediate stages of nasute evolution; gray- hydrostatic pressure; orange- muscular squeezing; purple, pistontype action. 